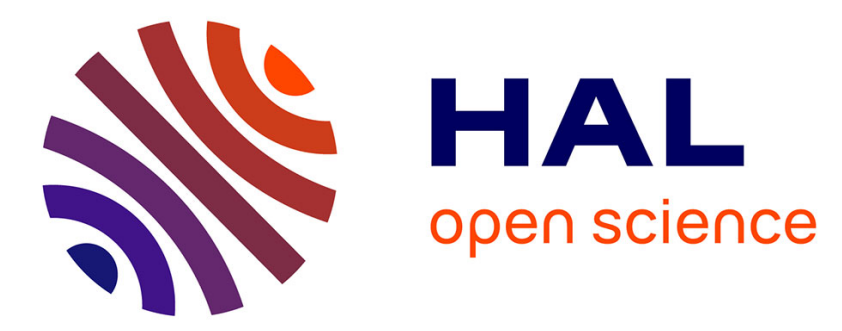

\title{
Lire en guerre. La lecture de la presse chez les combattants français entre 1914 et 1918 \\ Benjamin Gilles
}

\section{To cite this version:}

Benjamin Gilles. Lire en guerre. La lecture de la presse chez les combattants français entre 1914 et 1918. Guerres mondiales et conflits contemporains, 2012, 247 (3), 10.3917/gmcc.247.0007 . hal01793425

\section{HAL Id: hal-01793425 \\ https://hal.science/hal-01793425}

Submitted on 16 May 2018

HAL is a multi-disciplinary open access archive for the deposit and dissemination of scientific research documents, whether they are published or not. The documents may come from teaching and research institutions in France or abroad, or from public or private research centers.
L'archive ouverte pluridisciplinaire HAL, est destinée au dépôt et à la diffusion de documents scientifiques de niveau recherche, publiés ou non, émanant des établissements d'enseignement et de recherche français ou étrangers, des laboratoires publics ou privés. 


\section{Lire en guerre. La lecture de la presse chez les combattants français entre 1914 et 1918}

En novembre 1916, l'officier chargé du contrôle postal de la $130^{\text {ème }}$ Division d'infanterie consigne dans son rapport hebdomadaire une partie des propos désabusés qu'un soldat adresse à ses proches : «rien à lire, on est comme des bêtes, on a même pas les journaux »1.

Le motif, l'absence de lecture, peut sembler anodin en temps de guerre au regard des privations plus importantes, mais il paraît néanmoins suffisamment grave pour être signalé. En révélant l'état moral des hommes, la plainte éclaire sur la fonction centrale de la lecture dans les tranchées. Sa place est indirectement suggérée par le registre d'expression, qui voit les combattants se comparer à des animaux et conduire certains d'entre eux à employer le terme «d'ensauvagement $»^{2}$ pour décrire les conditions nouvelles de la guerre. Lire les journaux serait alors un moyen de lutte contre le processus de déshumanisation. Mais cette fonction civilisatrice constitue-t-elle l'unique raison qui amène les soldats à s'intéresser à la presse ? L'interrogation conduit à se pencher sur les différentes finalités de la lecture des périodiques au front et invite à réexaminer plus largement la réalité des pratiques. Comment en effet expliquer que d'un côté les journaux soient l'objet d'un fort rejet en raison de leur propagande outrancière et que, de l'autre, on puisse se plaindre de leur absence lorsqu'ils ne sont pas distribués?

Cette situation paradoxale a été mise en lumière pour l'arrière il y a près de vingt ans mais n'a pas été explorée depuis ${ }^{3}$. L'historiographie française de la Grande Guerre, à la différence de la recherche anglo-saxonne ${ }^{4}$, a consacré des très peu de travaux à la question de la réception. Elle s'est plutôt intéressée à la situation vécue par l'imprimé ${ }^{5}$ ou sur les conditions et les intentions de la production littéraire en temps de guerre ${ }^{6}$. Cette absence est d'autant plus remarquable que la lecture constitue depuis les études pionnières de Roger Chartier un objet

1 Service historique de la Défense (SHD), 16 N 1391. Contrôle de la correspondance de la $130^{\text {ème }}$ DI. Rapport du 15 novembre 1916.

2 C'est l'expression employée par J. Isaac dans sa correspondance. Jules Isaac, Un historien dans la Grande Guerre : lettres et carnets, 1914-1917, Paris, Armand Colin, 2004.

3 Stéphane Audoin-Rouzeau, «La notion de 'bourrage de crâne' en 1914-1918 : quelques éléments pour une remise en cause », Bulletin du Centre d'Histoire de la France contemporaine, Université de Paris XNanterre, $\mathrm{n}^{\circ} 8,1987$, p. 47-57.

$4 \quad$ Wolfgang G. Natter, Literature at war, 1914-1940. Representing the "time of greatness" in Germany, London, Yale University Press, 1999 et Robert Nelson, German soldiers newspapers of the First World War, Cambridge, Cambridge University Press, 2011.

$5 \quad$ Olivier Forcade, La censure politique en France pendant la Grande Guerre, Thèse de doctorat, Université Paris X-Nanterre, 1999.

$6 \quad$ Nicolas Beaupré, Les écrivains français et allemands de la Grande Guerre (1914-1920), essai d'histoire culturelle, Thèse de doctorat, Université Paris X-Nanterre, 2002. 
privilégié de l'histoire culturelle. Un regard sur les pratiques d'avant-guerre montre l'intérêt de suivre le phénomène jusque dans les tranchées.

La lecture est au cœur de la «culture médiatique ${ }^{7}$ qui caractérise la France de 1914, et c'est particulièrement vrai pour la presse : 10 millions de journaux sont vendus quotidiennement pour une population de 40 millions d'habitants ${ }^{8}$. Ce constat pose une question majeure : comment ont évolué les usages au contact de la guerre ? Deux types de sources apportent une réponse à cette interrogation. Celles du Ministère de la Guerre éclairent sur la place occupée par la lecture dans l'Armée et celles provenant des combattants eux-mêmes qui ont relaté dans leurs écrits leur expérience de lecture au front. Ces deux corpus permettent d'établir le cadre de fonctionnement de la lecture au front, et d'appréhender les pratiques développées par les soldats.

\section{Encadrer les pratiques de lecture au front : limites et tentatives}

La lecture au front s'exerce à l'intérieur d'un cadre spatial identifié et borné, la zone des armées. Ce territoire suit la ligne de front et couvre une profondeur d'environ 100 kilomètres. Il est soumis à l'étroit contrôle de l'Etat-major qui réglemente les différents aspects de la vie et des activités qui s'y déroulent. La circulation de l'imprimé n'échappe pas à l'exercice de ce pouvoir. Le commerce des livres et des revues est l'objet d'une étroite surveillance. Le commandement y interdit ainsi la vente de publications en dehors des lieux prévus et surveillés à cet effet. Deux types de point de vente se maintiennent, les librairies et les cantines pour les soldats, alors que le colportage disparaît. L'attitude française est assez restrictive si on la compare avec celle des autorités militaires allemandes. Celles-ci laissent s'installer sans trop de contrainte les libraires ${ }^{9}$. Le comportement français illustre une certaine méfiance pour la production culturelle, soumise à la censure par le décret du 5 août $1914^{10}$. Ce dispositif assure un contrôle unique et déconcentré des imprimés amenés à être diffusés sur le territoire, zone des armées incluse. Mais cette mesure du temps de guerre n'annule pas pour autant une disposition militaire plus ancienne, qui prévoyait le contrôle des lectures dans les régiments. En effet, le décret de 1913 relatif au corps de troupe n'est pas abrogé et continue de s'appliquer. Or ce document stipule que les officiers peuvent interdire les lectures lorsque

\footnotetext{
$7 \quad$ Jean-François Sirinelli et Jean-Pierre Rioux, La culture de masse en France de la Belle Epoque à aujourd'hui, Paris, Fayard, 2002, p. 14.

8 Christophe Charle, Le siècle de la presse : 1830-1939, Paris : Edition du Seuil, 2004, p. 137.

$9 \quad$ Wolfgang Natter, Op. cit., p. 34

$10 \quad$ Olivier Forcade, Op. cit., p. 10.
} 
celles-ci présentent une menace au maintien de l'ordre dans les unités ${ }^{11}$. Une seconde censure laissée à l'appréciation des gradés existe ainsi de droit dans la zone des armées. Une absence de contrôle centralisé par les états-majors introduit toutefois des différences d'appréciation selon les unités et ouvre ainsi à des abus d'autorité. Cela se produit au sein de la $8^{\text {ème }}$ D.I. en mai 1915. Le chef de bataillon du $130^{\text {ème }}$ R.I. interdit aux hommes de sa compagnie la lecture de titres de presse autres que les journaux populaires peu suspects (L'Echo de Paris, Le Journal, Le Matin, Le Petit Parisien, Le Petit Journal) et de droite, Le Figaro et Le Temps ${ }^{12}$. Ces excès trouvent malgré tout une limitation dans l'organisation même de l'armée. Même en guerre, l'armée demeure une entité composée de citoyens qui n'hésitent pas à recourir aux institutions républicaines pour dénoncer ces dérives. Des soldats se plaignent de ces pratiques auprès des représentants de la Chambre, lesquels s'emparent de ces faits, mettent en difficulté le Ministre de la Guerre en 1915 et 1916 et l'obligent à enquêter ${ }^{13}$.

L'action du pouvoir civil produit ses effets. Le GQG diffuse plusieurs notes ordonnant la cessation de ces interdictions arbitraires. La répétition de ces injonctions entre 1915 et 1917 montre la difficulté à faire appliquer la décision, jusqu'à ce que les Ministères de la Guerre et de l'Intérieur instaurent légalement en $1917^{14}$ un contrôle des imprimés à l'entrée de la zone des armées. Cette mesure introduit de droit une seconde censure qui vient se superposer à celle existante depuis août 1914 et qui s'applique à l'ensemble du territoire, zones militaires incluses. Dans ces espaces, les ventes et les abonnements à destination pourront donc être interdits par l'Etat-major. La décision de 1917 dépouille au passage les officiers de contact de leur maittrise des pratiques culturelles de leurs hommes au profit d'une centralisation de l'interdiction. Celle-ci se veut une réponse aux gestes de mauvaise humeur, de contestation et de refus qui se multiplient depuis l'hiver 1916. Assurer un contrôle uniforme et donc égalitaire des journaux qui parviennent au front doit ainsi contribuer à ramener la confiance des troupes dans leur commandement. L'instauration de cette mesure a produit une documentation qui renseigne aujourd'hui sur les ventes ainsi que sur les titres interdits et les volumes saisis.

\section{Evaluer la pénétration de la presse}

\footnotetext{
$11 \quad$ Décret du 25 août 1913 portant règlement sur le service intérieur des corps de troupe. Article 78. 12 SHD, 5 N 345, IV ${ }^{\text {ème }}$ Corps d'Armée, $8^{\text {ème }}$ DI, $130^{\text {ème }}$ RI, $1^{\text {er }}$ bataillon ; Note du chef de bataillon. 25 maI 1915.

13 SHD, 5 N 346, Ministère de la guerre, cabinet du sous-secrétaire d'Etat. Note pour le cabinet militaire, 8 mars 1917.

145 N 346, Note du Ministre de la guerre à Monsieur le Ministre plénipotentiaire chargé de la direction générale des Relations avec la presse. 2 août 1917.
} 
Ces informations permettent donc aujourd'hui d'aborder avec précision la question de la réception. La distribution quotidienne de la presse s'effectuait de manière centralisée à l'intérieur de chaque corps d'armée. Les dépôts principaux enregistraient pour chaque titre le nombre d'exemplaires entrés dans la zone et vendus ensuite dans les différentes coopératives. Les archives du II ${ }^{\text {ème }}$ corps d'armée sont à ce sujet très instructives ${ }^{15}$. Entre le 27 novembre et le 26 décembre 1916, arrivent en moyenne chaque jour 11280 exemplaires pour 40000 soldats. Ils sont vendus dans $90 \%$ des cas environ. Le rapport est d'un journal pour quatre soldats, ce qui est très voisin des ratios connus pour l'avant-guerre. Le volume des ventes permet de mesurer plus finement la diffusion de chaque titre. Cette évaluation a été possible pour le II ${ }^{\text {ème }}$ corps pour la période du 27 novembre au 26 décembre 1916. Le graphe ci-après prend uniquement en compte les ventes réalisées à partir des journaux arrivés dans les deux dépôts principaux du C.A, Proyart et Dampierre.

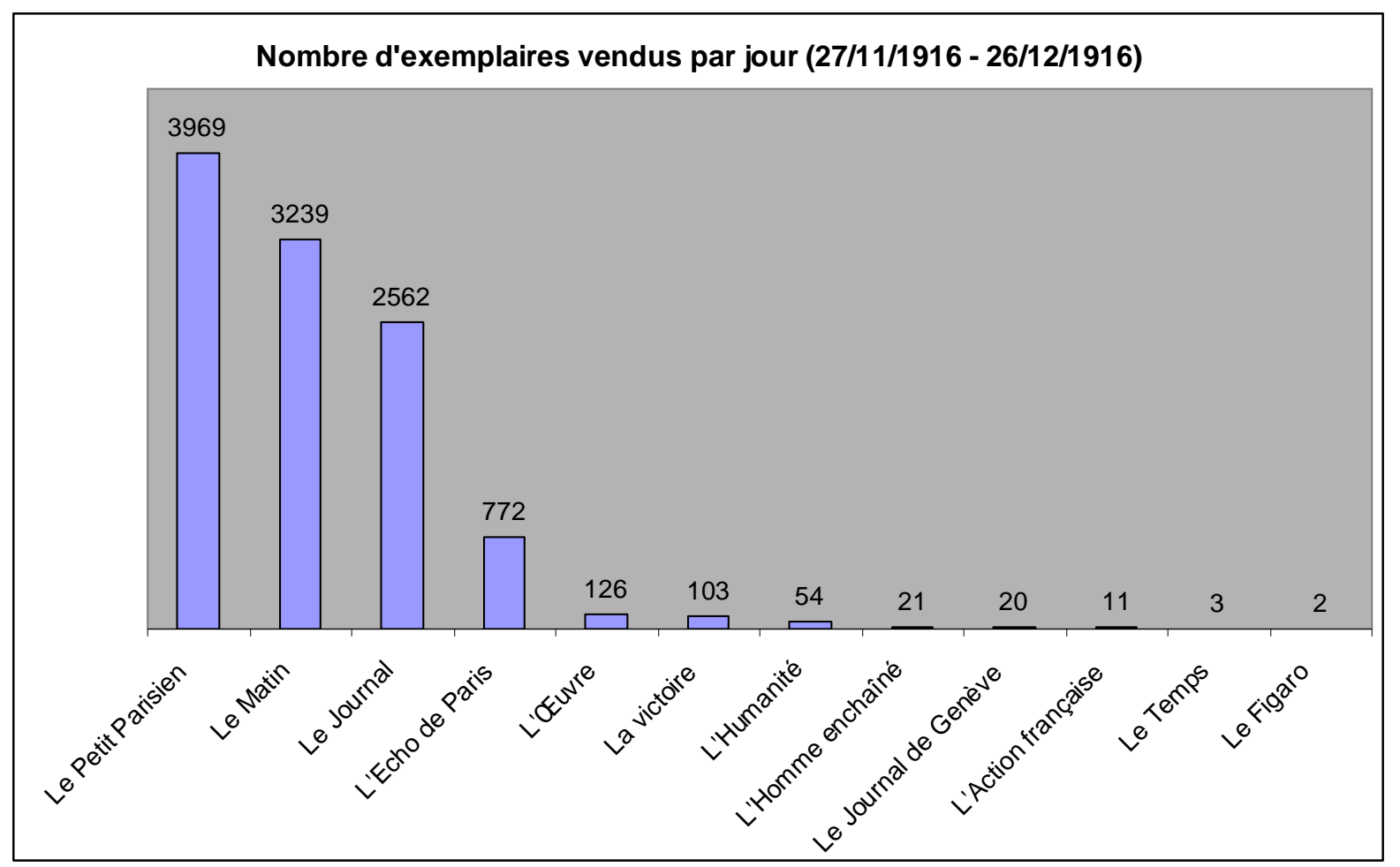

Le tableau appellerait plusieurs analyses qui dépassent le cadre de notre propos. Notons seulement que la presse la moins critique à l'égard de la conduite de la guerre représentée par les quatre grands populaires (Le Petit Parisien, Le Matin, Le Journal et L'Echo de Paris) est la plus lue. En généralisant ces données à l'ensemble des corps d'armée, il n'est pas imprudent d'estimer à 400000 le nombre d'exemplaires vendus chaque jour sur le front. Cette

15 SHD, 22 N 46, Bordereaux quotidiens de vente des quotidiens nationaux. Période du 27 novembre eu 26 décembre 1916. 
évaluation s'inscrit dans la temporalité de la guerre : la diffusion des journaux est inexistante jusqu'à la stabilisation du front en octobre-novembre 1914 et s'accroît très rapidement dès la fin de l'hiver 1914-1915 ${ }^{16}$. La lecture de la presse représente donc un phénomène de masse, attesté par les archives des autres corps d'armée ${ }^{17}$ et par les correspondances. Celle du capitaine Jules Jeanbernat donne une idée de la présence de l'imprimé : «vous ne sauriez. croire le nombre de journaux reçus au régiment. Si on les réunissait, il pourrait fournir une salle de lecture bien composée $\gg^{18}$. L'évaluation n'intègre pas la presse régionale, dont les envois ne peuvent être quantifiés, et la diffusion de la presse par colis. Il n'est possible que d'en affirmer l'existence à partir par exemple de la correspondance de Maurice Pensuet qui reçoit régulièrement par ce moyen-là les Annales politiques et littéraires ${ }^{19}$.

\section{L'impossible censure}

Cette masse de publications régulières envoyées quotidiennement dans la zone des armées complique les contrôles et limite l'efficacité des saisies. Malgré la centralisation des services postaux en arrière du front qui facilite en principe l'exécution de la censure, l'armée doit s'adapter à une situation à laquelle elle n'est pas préparée. Son attitude face au Miroir, journal illustré créé peu avant 1914, rend manifeste ces hésitations. La nouveauté du contenu, l'image photographique, perturbe les commissions de censure, habituées à l'analyse des textes mais moins à l'aise avec les publications illustrées. La difficulté à apprécier la signification de ces représentations entraîne une plus grande permissivité à l'égard de cette presse qui connaît une très large diffusion, y compris au front. Ce relâchement de la censure est entretenu par les rédactions qui pressent souvent les commissions à donner rapidement leur visa voire à passer outre les demandes de retraits ${ }^{20}$. Ces stratégies développées par les journaux invitent à nuancer l'idée d'un contrôle étroit et total des publications.

Le nombre des combattants présent et circulant dans la zone des armées limite également la portée des interdictions. Nombreuses sont les correspondances et les archives à exposer les tentatives de contournement mises en place par les soldats pour accéder à la presse. À la différence des lettres et des abonnements expédiés vers le front qui peuvent être saisis, les

1616 N 1473, Le télégramme du 25 février 1915 adressé par le Directeur du centre postal militaire au Ministre de la Guerre fait état de la réception de 3350000 lettres, 12000 journaux et 295000 paquets.

$17 \quad$ Les archives du XVIIe Corps d'armée (22 N 1210) analysent de manière plus précise le volume des ventes, La périodicité des publications est distinguée. Pour le mois de mars 1918, 31100 journaux sont vendus chaque jour.

18 Jules Jeanbernat, Capitaine Jules Jeanbernat Barthélemy de Ferrari Doria : Lettres de guerre (1914-1918), Paris, Plon, 1919. Lettre du 7 mars 1915, p.117.

19 Maurice Pensuet, Ecrit du front : lettres de Maurice Pensuet, 1915-1917, Paris, Tallandier, 2010.

$20 \quad$ O. Forcade, Op. Cit., p.472. 
colis ne peuvent être ouverts. Cet ensemble représente en moyenne 280000 envois par jour en $1915^{21}$ et constitue un moyen pour recevoir livres et journaux en toute discrétion. Jean Norton Cru se procure de la sorte l'ouvrage pacifiste de Romain Rolland Au-dessus de la mêlée $e^{22}$. Edité à Genève par Ollendorf, l'ouvrage pacifiste n'est officiellement pas interdit car, en vertu d'accords diplomatiques, les publications des Etats neutres ne sont pas soumises à la censure. Mais les officiers de contact ne se privent néanmoins pas d'interdire l'ouvrage en raison de son contenu, ce qui explique le mode d'envoi particulier de l'ouvrage à Norton Cru. La presse étrangère circule également de manière libre au front dans des volumes qui restent encore à évaluer.

Toutefois ces journaux, en majorité suisses et espagnols, peuvent être saisis en raison de leur contenu défaitiste, ce qui donne alors l'occasion de quantifier précisément leur pénétration au front. Ainsi, la confiscation de 1604 exemplaires l'édition du 31 août 1917 du Journal de Genève $e^{23}$ est très proche du volume de saisie de L'Humanité opérée le $1^{\text {er }}$ août 1917 au cours de laquelle 1762 journaux sont récupérés. La lecture de la presse étrangère francophone est donc loin de représenter un phénomène marginal. Elle démontre chez les soldats l'intérêt pour une information moins soumise à la propagande et exprime peut-être aussi la recherche d'un autre regard sur le conflit. Cette faculté à se soustraire au discours autorisé inquiète peu le Ministère de la Guerre jusqu'en 1917. L'accès à l'écrit, même séditieux, reste donc largement possible au front, comme le montre les différents moyens de contournement évoqués ici. Ainsi, l'Etat-major prend progressivement conscience au contact de la guerre de l'impossibilité de limiter la diffusion de l'imprimé, constat qui va l'amener et à opter pour une autre approche.

\section{Vers un encadrement des pratiques}

Les documents rédigés par le Quartier Général montrent un intérêt croissant à partir de 1916 pour la lecture. Elle devient en effet un enjeu de la mobilisation des hommes dans un contexte où la durée de la guerre impose de repenser les formes d'adhésion et de participation au combat. Ainsi les moyens mis en œuvre en 1914-1915 s'épuisent et doivent être renouvelés. L'échec du Bulletin des Armées de la République, créé en août 1914, illustre parfaitement ce cas. Le quotidien, qui se veut l'héritier éponyme de la publication de 1792, est un organe

\footnotetext{
$21 \quad$ SHD, 16 N 1473, Le télégramme du 30 janvier 1915 envoyé par le centre postal fait ainsi état de la réception de 290000 paquets à destination du front.

22 Archives municipales de Marseille, 46 II 4, Lettre du 6 octobre 1924 (A Alice).

23 SHD, 16 N 1562, GQG. Bordereau de saisie en date du 21 septembre 1917 : saisie de 1604 exemplaires du Journal de Genève.
} 
d'information destiné aux soldats. Ses articles sur la vie au front et sur les opérations militaires ont pour ambition de substituer le journal à la presse diffusée à l'arrière ${ }^{24}$. Or cette intention ignore complètement les pratiques de lecture des mobilisés héritées de l'avant-guerre et perpétuées au front. Cette méconnaissance de la réalité entraînera d'ailleurs sa disparition en 1917. L'Etat-major va alors explorer d'autres voies pour remobiliser les troupes.

Plus préoccupé par la presse de l'arrière, il va d'abord laisser se diffuser, tout en contrôlant le contenu, une presse artisanale écrite par et pour les combattants au sein des unités. Ces journaux de tranchées commencent à apparaître sur le front à la fin de l'année 1914. Leur contenu use souvent de l'humour et de l'ironie pour évoquer la situation des soldats, leur quotidien et leurs attentes. Leur critique se concentre en particulier sur les représentations fausses qui circulent dans les quotidiens à l'arrière ${ }^{25}$. Cette prise de position renforce le paradoxe d'une presse volontiers vilipendée mais pourtant très lue. Les conditions de fabrication de ces titres du front interdisent d'abord une diffusion large. La multiplication du nombre de journaux à partir de 1916-1917 et la mécanisation de la production accroissent très probablement leur pénétration parmi les troupes. Mais en l'absence de données relatives au tirage ou à la vente, leur diffusion demeure impossible à déterminer. C'est pourquoi nous ne les intégrerons pas dans la réflexion sur les pratiques de lecture.

A l'été 1916, Joffre autorise pour la première fois la presse à publier des récits glorieux mais réalistes de soldats. C'est une rupture avec la pratique antérieure où les seuls articles rédigés par des combattants étaient volontiers emphatiques. Il accorde également le droit de mentionner le nom des unités militaires engagées dans les opérations ${ }^{26}$. La nouveauté de ces mesures tient dans le lien qu'elles entendent créer entre les combattants et les civils. Il s'agit à la fois de renforcer la cohésion des troupes en stimulant la fierté régimentaire et d'impliquer les civils, désormais lecteurs des actions auxquels participent directement leurs proches. Cette ouverture s'accélère en mai 1917 avec l'arrivée de Pétain à la tête du GQG. Dès sa prise de fonction, il remet un rapport au Ministre de la Guerre consacré à l'influence de la presse au front $^{27}$. Ce document s'inscrit dans le contexte des mutineries comme une réponse immédiate à la contestation. Il s'insère aussi plus globalement dans un processus de mobilisation

SHD 5 N 565, Rapport du s/Lieutenant de Croisset, rédacteur en chef du Bulletin des Armées à Monsieur Lefort, chef adjoint du Cabinet de M. Le Président du Conseil [printemps 1917]

${ }_{25} \quad$ L'étude de ces journaux a été faite par Stéphane Audoin-Rouzeau. Stéphane Audoin-Rouzeau, Les soldats français pendant la guerre de 1914-1918 d'après les journaux de tranchées : une étude des mentalités, Thèse de doctorat, Université de Clermont-Ferrand, 1984.

265 N 332. GQG. Section presse. Note du 7 septembre 1916.

2716 N 271. GQG. Cabinet du commandant en chef. Note n²6204. 23 août 1917. 
culturelle qui touche la plupart des belligérants du front occidental ${ }^{28}$. Le rapport constate la réalité de la diffusion massive et généralisée de la presse dans la zone des armées. L'interdiction des journaux, bien que suspectés d'alimenter la contestation, comporterait trop de conséquences négatives. Le rapport préconise donc de mieux en encadrer le contenu, ce qui passe par la promotion de certains thèmes.

Le général en chef plaide ainsi pour la diffusion de thèmes patriotiques classiques, comme la reconquête de l'Alsace-Lorraine. Il prend également en considération un certain nombre de préoccupations exprimées par les combattants qui jusqu'alors n'étaient pas entendues par le commandant. L'évocation de la paix ${ }^{29}$ vient aussi briser un tabou et montre à elle seule le renversement d'attitude à l'égard de la presse : elle devient clairement pensée ici comme un levier à la mobilisation des hommes et à l'entretien de leur moral. Cette intention manifeste incite justement à ne pas réduire la notion de moral, utilisée dès 1915 par l'Armée, à une unique dimension de contrôle social. Elle est aussi un instrument d'étude de l'engagement des troupes, et c'est bien ce cadre analytique qui pousse Pétain à agir sur la presse. Alors que le Bulletin des armées de la République entendait remplacer en septembre 1914 toute la presse sur le front, le GQG intègre en 1917 la réalité des pratiques sociales et culturelles en vigueur dans la zone des armées. Le rapport de Pétain traduit donc l'adaptation à une situation où l'interdiction n'est pas possible et où d'autres formes d'encadrement de la lecture doivent être mises en place. La création des bibliothèques du front en 1917 semble la démonstration la plus tangible de cette tentative de maîtrise de la diffusion de l'écrit.

\section{Les journaux, grands absents des bibliothèques du front}

Le contrôle de la propagation de l'imprimé devient une préoccupation majeure pour les ministères de l'Intérieur et de la Guerre à partir de la première conférence pacifiste de Zimmerwald en septembre 1915.C'est à cette date en effet que commence à se multiplier les brochures, tracts et libelles dénonçant l'Union sacrée et appelant à la paix. La menace contestataire conduit ainsi l'Etat-major à renforcer sa surveillance des points de vente de la presse dans la zone des armées et à notamment faire la chasse au colportage, dont le suivi pose problème. La volonté de maîtriser la chaîne de diffusion conduit le commandement à créer au front des bibliothèques pour les soldats. Un catalogue de 2000 titres autorisés est constitué à cette occasion par une commission composée de civils (universitaires, membres du

28 Voir pour les cas des armées allemandes et britanniques : Alexander Watson, Enduring the Great War. Combat, morale and collapse in the German and the British Armies, 1914-1918, Oxford, Oxford University Press, 2008, p.81 notamment.

$29 \quad 16$ N 271. Ibid.,. 
Ministère de l'Instruction publique) et de militaires. Mais il ne comporte aucun titre de journal et de revue. Pourquoi ces lieux proposent-ils seulement des livres?

Dès leur création dans les années 1880-1890, ces espaces de sociabilité et de diffusion culturelle étaient souvent abonnés à de nombreux titres. Cette présence ne faisait que refléter l'essor des revues spécialisées en France à la fin du XIX ${ }^{\text {ème }}$ siècle et le travail des sociétés philanthropiques qui oeuvraient à la diffusion de la lecture dans les casernes. Ces actions ne disparaissent pas avec le déclenchement du conflit et suppléent l'absence de prise en charge de la lecture par le Grand Quartier Général. La Société Franklin, très investie avant guerre dans le développement des bibliothèques, envoie 32400 publications illustrées au front en $1915^{30}$. En 1915 toujours, l'oeuvre «Les foyers du soldats » crée ses premières salles de détente et de lecture dans les villes d'étape et dans la zone des armées. Néanmoins, ces structures mettent uniquement à disposition des livres et ne proposent pas de journaux.

Cette absence dans les fonds se comprend aisément : sa diffusion directe, par vente ou par abonnement, assure aux journaux une distribution rapide, la plupart du temps quotidienne, tant à l'arrière des lignes que dans les tranchées. Installées à plusieurs kilomètres du front et accessibles seulement lors des périodes de repos, les bibliothèques ne peuvent pas concurrencer ce mode d'envoi et d'achat qui correspond à un besoin d'accès immédiat à l'information. Acheter un quotidien pour le lire 6 voire parfois 8 jours après sa publication n'a en effet pas grand intérêt. Cet intérêt pour une information fraîche et renouvelée explique que les envois de périodiques faits par la Société Franklin passent de 32400 exemplaires en 1915 à 10000 en 1917. Il démontre également que la guerre n'a pas fondamentalement modifié les fondements culturels et sociaux de la société médiatique française. Bien qu'étroitement contrôlés et très critiqués par les combattants pour ce motif, les journaux continuent à jouer un grand rôle au front. L'attitude des soldats n'est donc pas aussi univoque que ce que semble l'admettre l'idée communément admise d'une presse vecteur du bourrage de crâne. L'examen des pratiques de lecture amène en effet à nuancer cette vision.

\section{La lecture au front : attitudes et pratiques}

\footnotetext{
30 Société Franklin pour la propagation des bibliothèques populaires et des bibliothèques de l'armée, Compte rendu de la séance tenue le 22 mars 1874, Discours de M. Ed. de Laboulaye sur l'éducation du pays par l'Armée, Paris, Librairie Ch. Delagrave, 1874, p. 42.
} 
Etudier l'attitude des soldats face à la presse n'est toutefois pas sans présenter de contrainte. Celle des sources se pose d'emblée. Les enquêtes menées dans les différents corps d'armée et qui alimente les propositions du général Pétain n'ont pas été conservées. Il n'est donc pas possible de savoir comment elles ont été menées ni sur quels groupes. Cette absence impose alors de se tourner directement vers les acteurs et de la lecture, les hommes mobilisés sur le front. Ces témoignages constituent le matériau unique pour appréhender les modalités de la réception des journaux au front. Aujourd'hui, cette documentation publiée représente un corpus très abondant. L'édition des récits directs, c'est à dire écrits pendant le conflit au plus près de la mémoire des événements, dépassait légèrement le millier d'ouvrages en $2011^{31}$. Tous n'évoquent bien sûr pas la question de la lecture au front, mais tous reflètent les pratiques culturelles et sociales de leurs auteurs ${ }^{32}$, souvent héritées de l'avant-guerre. Il faudrait ainsi pour chaque lecteur reconstruire l'itinéraire des pratiques pour comprendre la manière dont la guerre les façonne ou pas. Cette temporalité de l'acte de lire constitue une limite majeure à l'appréhension complète du phénomène. Mais elle n'empêche pas de cerner la question de la réception. En s'appuyant sur les expériences de lecteurs et en croisant les différents usages, il est possible de mettre au jour certaines pratiques communes et partagées.

La première est très certainement l'intérêt non démenti par les combattants pour la presse pendant toute la durée du conflit. Son attrait s'observe à travers les ventes, mais il s'agit d'un état, à un moment donné. La comparaison des tirages de journaux entre 1912 et 1917 éclaire sur une autre dimension, celle d'une croissance de sa lecture. Alors que le tirage de la presse nationale atteint 5,5 millions d'exemplaires par jour en novembre 1912, il grimpe à 6,5 millions en $1917^{33}$. Même si cette mise en perspective concerne tout le territoire, il n'en reste pas moins qu'elle décrit une tendance qui s'applique très certainement à la zone des armées. L'importance de la distribution au front le laisse fortement à penser. Cette progression des journaux s'accorde mal avec l'idée généralement admise d'une propagande verticale, complètement subie et rejetée par l'opinion publique et par les soldats. L'exemple des ventes au sein du II ${ }^{\text {ème }}$ corps d'armée et la plainte du soldat de la $130^{\text {ème }}$ DI notée plus haut viennent contredire cette perception de la presse. Lire n'est pas imposé par le commandement et n'est pas plus soumis à une quelconque pression sociale ou culturelle. C'est un acte librement

\footnotetext{
$31 \quad$ Cette estimation a été faite dans le cadre de notre doctorat à partir du dépouillement de quatre catalogues (Bibliographie nationale, catalogue informatisé de la BNF, catalogue du SUDOC et catalogue thématique de la BDIC). Nous avons comptabilisés 1165 récits de guerre publiés entre 1914 et 2011.

32 Jean Norton Cru avait montré en 1929 dans Témoins que le témoignage de guerre était surtout investi par les milieux culturels et sociaux supérieurs. Jean Norton Cru, Témoins, Essai d'analyse et de critique des souvenirs de combattants édités en français de 1915 à 1928, Paris, Les Etincelles, 1929, p. 666.

33 Archives nationales, F/7/12842, Préfecture de police, Cabinet du préfet, ler bureau.
} 
consenti porteur d'intentions. Quelques témoins ont entrepris cette réflexion sur l'acte de lire dans les tranchées, sur ses raisons et ses motivations. Léon Werth est probablement un des rares combattants à aborder en profondeur ces questions ${ }^{34}$, même si l'évocation de la lecture des journaux est commune à de nombreux récits et s'avère fondamentale à la compréhension de ces pratiques.

\section{L'adaptation à la guerre}

Le témoignage de Robert Dubarle se révèle être aussi extrêmement précieux pour saisir les contraintes de la lecture au front. Les conditions de vie dans les tranchées limitent les possibilités de lire. Elles bouleversent les pratiques, et en premier lieu le temps qu'il est possible d'y consacrer ou encore la concentration qui lui est nécessaire. Les formes nouvelles de la guerre, que ce soit le bombardement continu, le déroulement permanent des activités militaires permanentes ou encore l'exiguïté des lieux et la vie en communauté, contingentent fortement l'usage de la presse. Cette lecture se reporte sur les moments d'inactivité et suit un véritable rituel quotidien, comme l'exprime le capitaine Dubarle : «à 2 heures, je rentre. Le ravitaillement arrive; distribution de vivres ; il y a toujours un journal pour moi et souvent des lettres. [...] A 4 heures et demie la nuit tombe, mes postes rentrent. [...] Je relis mon journal. Je fais un peu d'anglais. A 7 heures et demie chacun regagne ses pénates. Me voilà tout à fait seul, et jusqu'à 9 heures, je peux encore lire, écrire ou rêvasser $»^{35}$. Malgré le relatif confort dont il bénéficie, la lecture est fractionnée durant la journée et se déroule sur des plages de temps relativement courtes. Dans ce contexte, le découpage du contenu en articles est plus adapté à la vie au front. Dans le cas de Robert Dubarle, lire la presse ou un livre relève de deux pratiques différentes. La première s'inscrit dans l'espace social qu'est la tranchée alors que le second se pratique dans une sphère privée et intime. Les deux fonctions traditionnellement accordées à la lecture, publique et individuelle, ne sont pas fondamentalement transformées au contact de la guerre.

Dans un contexte où le déplacement des unités entre les zones de combat et de repos est fréquent, la presse est particulièrement bien adaptée à ce type de contrainte logistique. Le transport des journaux ne représente pas une surcharge pour les sacs ou un encombrement intolérable pour les besaces. Ils sont légers, pliables et leur consommation immédiate n'exige pas de condition de conservation particulière. Cette praticité renforce-t-elle la pénétration des

\footnotetext{
34 Léon Werth, Clavel soldat, Paris, Viviane Hamy, 2001. Toutes les références sont prises dans cette édition.

$35 \quad$ Robert Durbarle, Lettres de guerre de Robert Dubarle, capitaine au 68è bataillon de chasseurs alpins, Paris, Perrin, 1918, Lettre du 15 janvier 1915, p.100-101.
} 
quotidiens dans les tranchées? Le niveau comparable de lecteurs moyens de quotidiens entre l'avant-guerre et les années 1914-1918 incite à répondre par la négative. En revanche, la lecture profite très certainement de cette adaptation aux conditions de la guerre des tranchées. Les demandes d'envoi de livres, à l'image de celles de Dubarle ou de Norton Cru, insistent sur la nécessité de leur formats réduits et de leur faible encombrement. Ces attentes n'échappent pas aux éditeurs qui, conscients de disposer dans la zone des armées, d'un lectorat potentiel captif, s'adaptent pour proposer des séries spécialement destinées aux combattants. La création de la collection «La bibliothèque du front » en 1915 par la maison d'édition Payot, montre l'intégration de ces contraintes : les ouvrages ne dépassent pas les 200 pages et leurs dimensions sont très proches de nos livres de poche Là encore, l'initiative n'est pas complètement nouvelle. Elle s'inscrit dans un processus de transformation du livre en objet de consommation courante entamé depuis la fin du $\mathrm{XIX}^{\mathrm{ème}}$ siècle. La guerre accélère une évolution culturelle et sociale pour la presse.

\section{Endurer la guerre}

La large diffusion des journaux n'est pas seulement à chercher dans leur praticité mais dans leur contenu. Ce sont les informations qu'ils contiennent qui guident l'intérêt des soldats. Les quotidiens, entre 1914 et 1918, concentrent la diffusion des informations autour de deux thématiques : la vie quotidienne à l'arrière et la guerre. Ces deux axes constituent pour les soldats une forme de «trop vide et de trop plein ». Coupés physiquement et spatialement de l'arrière, ils vivent une réalité complètement différente de celle des civils. Immergés dans la guerre, ils la subissent par son omniprésence. Pourtant, ce sont ces deux raisons qui expliquent le succès de la presse au front. D'abord, comme l'indique Léon Werth dans un passage de Clavel soldat, lire le journal relie le lecteur à un autre monde : «Vernay et Clavel lisent cela dans leur trou. Et ce style de journal, en temps de paix, les eut fait sourire simplement. Mais ce journal vient de Paris, du monde presque miraculeux où il y a des maisons avec des toits, du monde où persiste la diversité des êtres, du monde où la vie et la mort sont des événements ${ }^{36}$. L'acte de lire rattache à un arrière vu, par opposition au front, comme un monde civilisé. Cette perception contribue à entretenir le sentiment d'exceptionnalité de la guerre. En permettant de connecter ces deux mondes, les journaux fournissent, en déplaçant la réalité vers l'arrière, une stratégie de survie. Le front, lieu d'intense déshumanisation, conserve ainsi sa dimension extraordinaire et anormale. Par l'acte

36

Léon Werth, Op. cit., p. 109. 
de lecture, le combattant considère ce dernier comme une parenthèse dans son existence, avant le retour à la vie. Lire aide à survivre et à tenir dans les tranchées et permet de croire en la possibilité d'un « après ».

Cette projection donne corps à un retour à la normalité et permet aussi au lecteur de s'imaginer sortir vivant de la guerre. C'est pourquoi l'intérêt pour les communiqués de l'Etatmajor, les articles de journaux publiés lors des offensives ou encore les actions militaires à l'étranger relatées dans la presse est si grand chez les mobilisés. Edouard Coeurdevey passe un temps certain à commenter dans son journal les aspects internationaux du conflit et l'entrée en guerre de l'Italie. Il en est informé par les différents quotidiens auxquels il a accès. C'est pour lui un moyen d'envisager une fin rapide des hostilités. Jean Norton Cru et Maurice Pensuet manifestent une pareille attitude lors des offensives de Champagne de 1915, de la Somme et même du Chemin-des-Dames. Tous trois attendent, à travers leur lecture, une percée qui ouvrira à la victoire. A chaque offensive perçue comme décisive, et c'est particulièrement vrai chez Norton Cru, la croyance dans la victoire se remobilise. Lire s'inscrit ici dans une attitude dominée par l'espoir d'une issue prochaine et heureuse de la guerre, où se croisent un désir personnel d'en réchapper et un aspiration collective d'ordre patriotique.

\section{Donner sens à l'expérience des tranchées}

La lecture de la presse contribue à créer un double horizon d'attente et à borner deux temporalités. Celle qui succède à la guerre, mais également celle du présent. Dans ce cadre, lire revêt une autre finalité, plus immédiate, exprimée par Léon Werth : «sortant de la boue où ils [les hommes] n'ont rien entendu que des éclatements et des sifflements, où ils n'ont rien vu que des trous et des fossés, où ils ont pataugé selon l'avance ou le recul du troupeau, ils racontent ce qu'ils ont lu, selon les récits des journaux. [...] Mais dans les récits des journaux, ils trouvent une explication. Ils la plaquent sur le froid, la boue, la nuit, les morts. Elle donne un sens logique à leurs pas hésitants dans cette glue faite de terre mouillée, d'excréments et de morts ${ }^{37}$. Lire donne sens à l'expérience vécue dans les tranchées car la pratique apporte de l'intelligibilité à un conflit dont la dimension spatiale, la violence et les cadres sensoriels dépassent la compréhension et la capacité à raconter.

La lecture des quotidiens permet d'exprimer et de verbaliser les impressions et les perceptions ressenties au front. Le récit journalistique opère une mise en forme des représentations et constitue de la sorte un moyen d'objectiver l'expérience de guerre. Il façonne et structure ce 
qui est vu et ressenti par ces hommes plongés dans l'indicible des tranchées. Les journaux créent une conscience de l'événement et cet usage malmène l'idée que les soldats auraient eu «du mal à se faire une idée cohérente de la guerre $»^{38}$. Au contraire, La transformation de la lecture en une expérience objectivée tend plutôt à démontrer que le contenu propagandiste des journaux est complètement intégré au sein d'une recherche de sens. Et c'est peut-être cette ambivalence qui donne la clé d'interprétation de l'importante diffusion de la presse au front. Elle permet de comprendre non seulement ce qui est quotidiennement vécu mais également ce qui se joue à une échelle spatiale plus large. De ce fait, elle replace le combattant dans la guerre et participe à légitimer son engagement présent.

Cette faculté à impliquer le lecteur dans le combat et cette propension à construire le sens de son engagement reposent sur une très large adhésion volontaire des troupes. Les titres les plus diffusés au sein des II ${ }^{\text {ème }}$ et XVII ${ }^{\text {ème }}$ corps d'armées - unités pour lesquelles nous disposons des renseignements les plus précis - sont ceux dont la ligne éditoriale est la plus marquée par le discours patriotique ${ }^{39}$. Quatre titres concentrent de manière quasi exclusive les ventes chez les marchands de journaux de la zone ou des coopératives : Le Petit parisien, Le Journal, L'Echo de Paris et Le Matin. Outre que leur diffusion au front confirme la popularité acquise avant 1914, ces journaux fournissent une explication au combat et apportent une motivation aux hommes. Ils répondent malgré leur propension à l'emphase patriotique aux questions que se posent les soldats. Ils participent ainsi à créer une identité commune propre à la tranchée. Le mode de lecture de la presse, souvent réalisée à voix haute au sein d'un groupe, cimente cette construction qui traduit un besoin de dépasser l'expérience personnelle de la guerre pour l'enserrer dans une représentation collective. C'est tout le paradoxe de la presse dans les tranchées entre 1914 et 1918, qui même si elle est critiquée et stigmatisée, joue un rôle capital dans la cohésion des troupes.

Ce regard neuf posé sur le phénomène de lecture de la presse au front invite à aborder de manière un peu différente les questions de contrôle, de culture de guerre et d'expérience combattante. L'attitude des autorités militaires françaises à l'égard des journaux n'a pas été monolithique, mais a progressivement évolué entre 1914 et 1918 passant de l'interdit à l'intégration du facteur presse dans la mobilisation des soldats. Cette mutation illustre la prise de conscience progressive de la réalité sociale et culturelle de l'imprimé, dont la guerre ne fait

\footnotetext{
38 Modris Eksteins, Le sacre du printemps : la Grande Guerre et la naissance de la modernité, Paris, Plon, 1991, p.206.

$39 \quad$ Claude Bellanger, Jacques Godechot, Pierre Guiral (dir.), Histoire générale de la presse, Tome III, De 1871 à 1940, Paris, Presses Universitaires de France, p.430-431.
} 
que confirmer l'omniprésence. Ainsi, il est fondamental pour comprendre l'usage des quotidiens au front ne pas rejeter et isoler les pratiques antérieures à 1914. La guerre n'introduit en effet pas de rupture dans l'acte de lire. Ces remarques amènent à considérer la lecture de la presse dans une chronologie étendue, qui intègre l'avant-guerre mais également les années 1920-1930. Il est difficile de ne pas percevoir la forte expansion des journaux chez les anciens combattants, et peut-être plus largement dans la société française, comme une conséquence directe des comportements rencontrés dans les tranchées. Une attitude qui invite aussi à nuancer l'idée d'une propagande verticale imposée par l'imprimé et qui aurait provoqué un très fort rejet.

Les ventes montrent un comportement inverse. Elles expriment plutôt qu'une forme d'acceptation de son contenu a plutôt prévalu au front. C'est que, malgré ses exagérations et ses outrances bien notées par les soldats, la presse a été considérée comme un vecteur d'identité et de sens commun. Dans ce cadre, elle a formé un remarquable outil d'homogénéisation de l'expérience combattante, qui explique pour parti l'endurance dans les tranchées. Lire a permis de comprendre la guerre, de donner sens et de justifier l'engagement individuel, au-delà des réserves ou des colorations politiques et idéologiques des titres. L'analyse des journaux les plus vendus dans la zone des armées en apporte une démonstration éclatante. La presse discutant la guerre, que ce soit du bout de la plume ou plus franchement comme La Vague, est diffusée dans des quantités infiniment moindres que la presse patriotique. Mais il ne faut pas en déduire non plus trop rapidement à une acceptation généralisée de la guerre et à ses buts politiques. L'attention soutenue des lecteurs aux annonces de victoire est ainsi surtout une stratégie de survie : elles apportent une raison pour tenir et s'accrocher. Aussi, l'acte de lire, et c'est peut-être sa fonction première, se révèle être un puissant instrument de lutte contre le processus de décivilisation qui caractérise l'univers des tranchées et, plus largement toute expérience extrême. Et il n'est pas absurde à ce propos de construire un parallèle entre l'attente du soldat de la $130^{\text {ème }}$ DI relevé par le contrôle postal et le déporté Georges Semprun. Dans son récit autobiographique L'écriture ou la vie, l'écrivain espagnol confère à la lecture la même finalité que celle exprimée par le fantassin en 1917 : celle du pouvoir de la lecture à maintenir l'individu dans l'humanité. 


\section{Résumé / Abstract}

\section{Lire en guerre. La lecture de la presse chez les combattants français entre 1914 et 1918}

La large diffusion de la presse quotidienne dans les tranchées vient nuancer l'idée communément admise d'un rejet massif de celle-ci par les combattants. Malgré la censure particulière qui s'exerce dans la zone des armées, les journaux sont lus dans des proportions très voisines à celles de l'avant-guerre. En 1914, l'Etat-major français a une attitude très méfiante à l'égard des usages de la presse. Mais progressivement, la lecture est intégrée dans sa stratégie de mobilisation. Cette évolution ne fait néanmoins qu'intégrer la réalité des pratiques de lecture chez les soldats, que le contact de la guerre n'a pas modifié pas en 
profondeur. En revanche, lire la presse dans les tranchées répondait à des attentes particulières et immédiates. Elle permettait de façonner l'expérience vécue, de donner sens à l'engagement des hommes et à légitimer socialement celui-ci.

\section{Read at war. Press reading in French Army during the Great War}

The large circulation of daily press in trenches leads to modify the usual idea of his rejection by French soldiers. Despite the censorship in the war zone, newspapers are read as much as they were before the war. In 1914, the French General Staff is very suspicious concerning the newspaper's reading. But progressively, the command understands the role of the press in the combatants mobilization. This progress is only considering the reality of the practices on the front. In this case, the Great War has no deeply changed usages. On the other hand, to read newspaper responds to specific and immediate needs : the reading is a way to build the trenches' experience and make their engagement truly meaningful.

\section{Eléments biographiques}

\section{Benjamin GILLES}

Doctorant en histoire à l'Ecole des Hautes Etudes en Sciences Sociales. (sujet de thèse : Lire en guerre, lire la guerre. Jean Norton Cru et la genèse de Témoins. Dir. S. Audoin-Rouzeau) Conservateur. Responsable à la BDIC du Département des périodiques 